\title{
Article \\ A Blockchain-Based Intelligent Machine Learning System for Smart Health Care
}

 \\ 1 Institute of Data Science and Digital Technologies, Vilnius University, LT-08412 Vilnius, Lithuania; \\ \{mir.hassan@mif.stud.vu.lt, remigijus.paulavicis@mif.vu.lt, ernstas.filatovas\}@mif.vu.lt \\ 2 Key Laboratory of Aerospace Information Security and Trusted Computing, Ministry of Education, School of \\ Cyber Science and Engineering, Wuhan University, Wuhan 430072, China; adnan@whu.edu.cn \\ * Correspondence: remigijus.paulavicis@mif.vu.it
}

\begin{abstract}
Blockchain and Machine Learning gives the best solutions together in performing various tasks in the Smart Health care system. With these two new emerging technologies, that have materialized in the last decade. In this paper, we proposed secure, transparent and intelligent methods in the Internate of medical things industry using Machine learning models and blockchain technology to enhance security level and train our models to improve diagnostic, prevention, treatment of the patient, patient rights, patient autonomy and equality in the health care system.
\end{abstract}

Keywords: Blockchain technology; security; Machine learning; Smart System

\section{Introduction}

Frost \& Sullivan expects that during the next five to ten years, healthcare companies will have access to integrated health IT platforms based on an emerging collection of information management technologies, such as blockchain [1], the Internet of Things (IoT) [2], and machine learning [3]. When it comes to storing and exchanging health data, privacy is a key problem, and with current healthcare data storage systems lacking top-tier security, blockchain could give a solution to vulnerabilities like hacking and data theft. Interoperability is a feature of blockchain technology in healthcare that allows for the secure interchange of medical data among the various systems and employees involved, resulting in a number of benefits such as improved communication, time savings, and operational efficiency. According to the report, due to difficulties such as errors, duplications, and inaccurate billing, the use of blockchain technology for claims adjudication and billing management applications is expected to expand by 66.5 percent by 2025 . With blockchain, all of these issues can be solved.

Machine learning is a computer-based method for analysing free-form text or voice

Citation: Hassan, M.; Paulavicius, R

Filatovas, E.; Iftekhar A. A

Blockchain-Based Intelligent Machine Learning System for Smart Health

Care. Preprints , 1, 0 .

https://doi.org/

Academic Editor:

Received:

Accepted:

Published:

Publisher's Note: MDPI stays neutral with regard to jurisdictional claims in published maps and institutional affiliations. using a preset set of theories and technologies, such as linguistic and statistical methodologies, to extract rules and patterns from the data. Knowledge and experience are two very significant variables for physicians in terms of patient care; yet, humans are limited in terms of gaining knowledge through accumulating data, whereas machine learning excels in this area [4]. There are two types of machine learning: supervised learning and unsupervised learning [5].

\section{Literature Review}

Because of the heightened danger of pandemic, the World Health Organization (WHO) has suggested that countries develop a "Pandemic Plan." A Pandemic Plan is often designed in accordance with the WHO's pandemic phases, with the goal of achieving unambiguous results in pandemic management from the start [6].

Different techniques to preparing for an emergency may be recognised in healthcare; in fact, each disaster is divided into four phases: mitigation, preparation, reaction, and recuperation [7]. The "tabletop exercise" is a valuable technique for simulating the creation of a crisis situation; it creates a scenario that benefits from both communication and cooperation among various sectors and areas, such as management, workers, logistics, 
communication, and finance. A proper method could give a broad framework as well as a mental model that replicates the ideal environment for future decision-making [7].

Each transaction performed in a block of the network is validated through a process based on the consensus distributed across all nodes (that is, the devices/users connected to the net). Blockchain technology belongs to the larger category of Distributed Ledger technologies, whose functioning is based primarily on a register structured in blocks linked in a network; each transaction performed in a block of the network is validated through a process based on the consensus distributed across all nodes (that is, the devices/users The transactions are the outcomes of the operations that take place among the network's subjects. The concept of blockchain comes from the fact that each block keeps a reference to the previous one using a cryptographic method. Blockchain is not stored on a centralised server like typical online services, but rather on network devices (computers) called nodes, each of which has a copy of the entire blockchain.

Furthermore, two important elements of this type of technology should be highlighted for our analysis: (i) decentralisation of consensus and (ii) decentralisation of ledgers. The existence of trustworthiness among the individuals participating in any form of transaction and the presence of a central authority may no longer be necessary due to the decentralisation of consensus [8][9]. Similarly to the second point, the replication and storage of different copies of different blockchains across network nodes ensures greater system security and equity among users, who can access the same information at the same time, and thus the traceability and immutability of the validated transactions contained in the blocks. As a result, blockchain is a peer-to-peer network in which all network users can trust the system without having to trust one another.

\section{Blockchain in Health Care System}

By keeping a specified set of standardised data on the chain, together with private encrypted linkages to separately stored information such as radiographic or other images, the blockchain might enable a single transaction layer where businesses can submit and share data through one secure mechanism. The usage of smart contracts and standardised authorization standards can greatly aid in the provision of seamless connectivity. Between 2009 and 2017, there were approximately 176 million data breaches involving healthcare records [10]. The blockchain's secure characteristics can significantly improve the security of health data. Each person has a public identification or key and a private key that can only be opened when and for the length of time required. Furthermore, the requirement to attack each user individually in order to obtain sensitive information would limit hacking. As a result, blockchains can provide an immutable audit trail of medical data. Figure 1 shows a diagram. For the health-care industry, we presented a blockchain management system.

\subsection{Tracing and securing Medical Supplies}

With complete transparency, blockchain can assist protect and identify the trail of pharmaceutical supply. It can even track the labour expenses and carbon emissions associated with the production of these items.

\subsection{Stroing Medical Information}

Though there has been substantial progress in the storage of medical reports, their transactions and security remain inadequate. Health data breaches are common, and with the rise of hackers all over the world, hacking of these data has become the most common technique of breaching, eclipsing previous methods. Meditab[11], a software firm, claims to be one of the leading electronic medical records software developers for hospitals. Electronic faxes are included in the company, and this way is still widely utilised to transmit patient data with other data seekers. However, this mode of data sharing has shown to be unsafe and unreliable, making it a poor choice. A fax server at Spider Silk [4], a Dubai-based cybersecurity outfit, was running an Elastic search database with at 


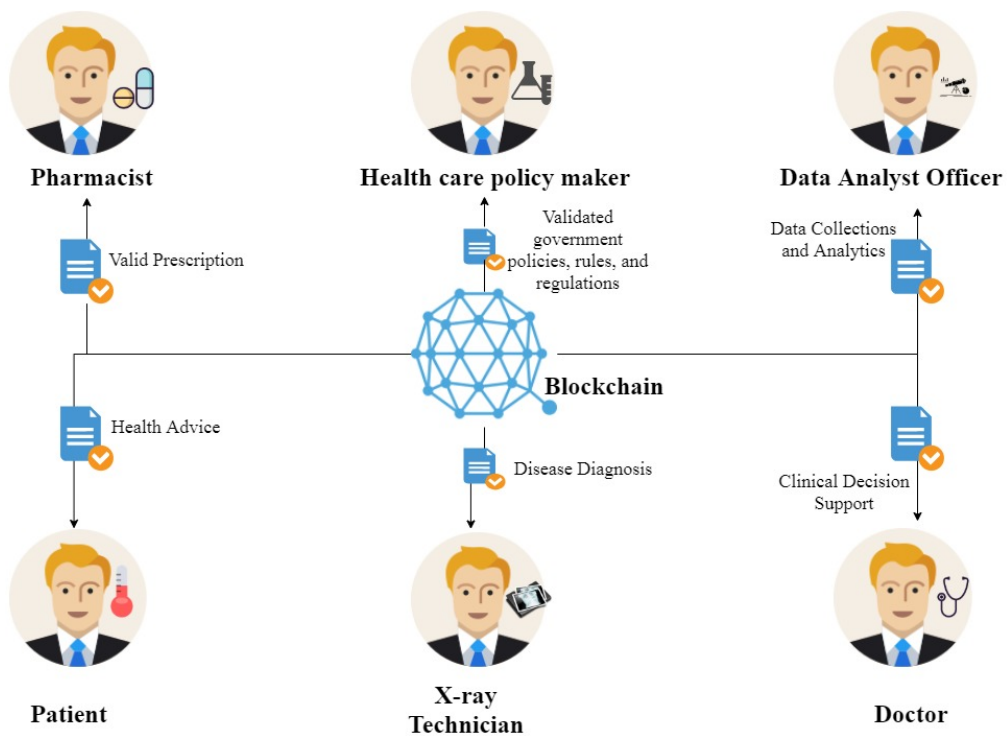

Figure 1. Blockchain Health Care Management System Overview

least 6 million health-care records. The server did not even have passwords for protection, allowing anyone to see the transmitted faxes in real time. The faxes transmitted contained a variety of identifiable information on the patients, including their medical history, previous treatment, Social Security numbers, and other records.

\subsection{Tracking Disease and OutBreak}

The unique characteristics of blockchain can aid real-time disease reporting and disease pattern investigation, which can aid in determining the disease's origin and transmission factors.

\section{Artificial Intelligence and Machine Learning in Health Care System}

The rise of contemporary computing technologies has been inextricably tied to Artificial Intelligence (AI). Machine learning has deep historical origins, with Alan Turing's work in deciphering the German Enigma machine during World War II serving as the foundation for most of modern computer science. He is also the subject of the Turing Test, which seeks to determine whether AI has become indistinguishable from human intelligence [5].

\subsection{Machine Learning in Health Care System}

In the medical area, machine learning, a branch of artificial intelligence, has been widely applied. Information such as patient information, medical treatment records, and medication status has been digitized, and a great quantity of data has been generated in the field of medicine and healthcare as a result of the advancement of ICT technology and the introduction of the era of big data [12]. Machine learning is being used in the medical business to analyze complicated medical data, which has become the main focus of machine learning research.

The study looks at how the medical business employs machine learning technologies including deep learning, neural network learning, and feature fusion to accomplish data analysis and mining. The goal is to realize human activity detection, health monitoring, disease prediction, and diagnosis, as well as advance the field of smart medicine.Gumaei et al. [13] used machine learning to recognize human activity. They proposed a multisensor hybrid deep learning model for human activity recognition. This approach can more intelligently process multisensor data, allowing medical institutions to better care for the elderly and ill. To continually monitor the physical status of students, Souri et al. [14] introduced machine learning to the student health monitoring system, collected data through the Internet of Things, and evaluated data using machine learning.Ali et al. [15] 
developed a disease prediction system based on deep learning feature fusion and the information gain technique to anticipate the occurrence of heart disease and generate conditions for effective treatment of heart disease patients. Chui et al. [16] examined recent research in the field of intelligent medicine on illness diagnosis, outlined new machine learning algorithms, and considered the obstacles of implementing disease diagnosis in the future.

\subsection{Supervise learning}

Supervised learning typically employs training data as labelled data. Training data has one or more inputs and has "labelled" output. These labelled findings are used by models to assess themselves during training, with the goal of improving new data prediction (i.e., a set of test data). Typically, supervised learning models concentrate on methods for classification and regression. In medicine, classification issues are fairly common. In most clinical settings, a clinician diagnoses a patient by identifying the illness based on a set of symptoms. Regression challenges usually focus on forecasting numerical outcomes, such as projected length of stay in hospital, based on a set of data, such as vital signs.

Random forests (RF), decision trees (DT), Nave Bayes models, linear and logistic regression, and support vector machines (SVM) are examples of supervised learning techniques, while neural networks can also be trained this way [17]. Random forests are a type of decision tree that is made up of a group of decision trees that have been trained individually. To produce a better end result and prediction, the trees' projections are often averaged [18]. Each tree is constructed using a random sample of the data with replacement, and a random subset of features is chosen at each candidate split. This keeps each learner or tree from focusing too heavily on the training set's ostensibly predictive properties, which may or may not be predictive on incoming data. In other words, it improves the model's generalisation. Random forests, which can include hundreds or even thousands of trees, operate well with noisy data [19]. The model that is built by combining the results of many trees trained on the data will produce a prediction that can be tested with test data [Figure 1]).



Figure 2. Architecture of Blockchain and Artificial Intelligence in Smart Healthcare System

\subsection{Unsupervised Learning}

Unsupervised machine learning uses unlabeled data to find patterns within the data itself [19]. These algorithms typically excel at clustering data into relevant groups, allowing for detection of latent characteristics which may not be immediately obvious. However, they are also more computationally intensive and require a larger amount of data to perform. K-means clustering and deep learning are the most well-known and widely utilised methods, however deep learning can also be applied supervised [12, 20]. Association tasks, which are comparable to clustering, are also performed by such algorithms. Because no human input is given as to which set of traits the clusters will be concentrated on, these 
algorithms are termed unsupervised. Although there are various modifications of the standard k-means algorithm, such as k-medians and k-medoids, the fundamental remains the same. In the case of kclusters, the technique uses Euclidean distance to locate the "nearest" centre or mean. The current data point is then assigned to that cluster, and the cluster's centre is recalculated, updating it for the next data point [21]. The most significant disadvantage of this technique is that it requires an expected number of "means" or "centres" to be initialised. Poor clustering can be caused by incorrect kvalue selection.

\section{Working Process}

The suggested architecture is intended to provide for safe administration of activities in the intelligent technological environment. These activities comprise system startup, sensors and actuator data collection, and reliable customer control over the device to perform desired operations. All these procedures are explained briefly in the following sections.

\section{Machine Learning Algorithms in Health Care Industry}

\subsubsection{Support Vector Machine}

The most common machine learning method utilised in the healthcare industry is the Support Vector Machine (SVM). For classification, regression, and detection outliners, it employs a supervised learning model. In recent years, algorithms have been used to forecast cardiac patient medication adherence, which has helped millions of people avoid dire repercussions including hospital re-admission and even death. Protein classification, picture segregation, and text categorization are all examples of applications. [17].

\subsection{Logistic Regression}

Using predictor variables, this machine-learning technique predicts the present scenario of the category dependent variable. It's frequently used for categorising and forecasting the likelihood of an event, such as illness risk management, which helps clinicians make critical decisions. It also assists medical institutions in identifying patients who are at higher risk and developing behavioural health strategies to help them improve their everyday health behaviours [17]. Mathematically it can be represented as:

$$
p=\frac{\exp \left(b_{0}+b_{1} x_{1}+b_{2} x_{2}+b_{3} x_{3}+\ldots \ldots .+b_{u} x_{u}\right)}{1+\exp \left(b_{0}+b_{1} x_{1}+b_{2} x_{2}+b_{3} x_{3}+\ldots \ldots .+b_{u} x_{u}\right)}
$$

The terms terms in the equation identifies as, expected probability $\mathrm{p}$, independent variables from and the regression coefficients are from $b 0+b 1$, further more it can be compressed as:

$$
\ln \left(\frac{p}{1-p}\right)=b_{0}+b_{1} x_{1}+b_{2} x_{2}+b_{3} x_{3}+\ldots \ldots+b_{u} x_{u}
$$

Defined equation is known as simple logistic regression model.

\subsubsection{Random Forest}

The approach is used to build several training trees at training time for classification and regression, as well as to overcome the problem of over-fitting decision trees. Random Forest is used to predict illness risk and to analyse ECG and MRI data based on a patient's medical history. [18].

\subsubsection{Discriminant Analysis}

Discriminant Analysis is a machine learning approach for evaluating the accuracy of object classification and assigning one object to one or more categories. Discriminant analysis is used in the healthcare business for everything from early detection of diabetic peripheral neuropathy to refining the diagnostic characteristics of blood vessel imaging. 
It's also utilised to detect indicators of mental health disorientation and for electronic health record management systems.

\section{Solution via Blockchain and Artificial Intelligence}

Healthcare accounts up a significant portion of the gross domestic product (GDP) in developed countries. Hospital expenditures, on the other hand, continue to rise, as do wasteful procedures and health data breaches. This is one area where blockchain technology has the potential to improve things. It is capable of a wide range of tasks, including secure encryption of patient data and the management of epidemics. Estonia is a pioneer in this arena, having implemented blockchain technology in healthcare in 2012. Currently, blockchain is used to maintain its complete healthcare billing system, 95 percent of health data, and 99 percent of prescription information [19]. In Figure 1, we will elaborate on the paradigm of Blockchain and Artificial Intelligence application in the health care system. Certificate Authority will provide an authorised certificate to each user in the Blockchain network. It will give the person who transacts in the network an identity. A digital certificate will serve as the identity. The user will sign the transaction and upload it to the blockchain using the digital certificate. . The benefits of signing are:

- Ensure that the user conducting or requesting any transaction is a legitimate user by verifying their identity on the blockchain.

- Ascertaining that the user has permission to access the ledger for the transaction at hand.

How blockchain can become a problem solver?

1. Every user that has been authenticated will have a copy of the shared ledger. This will address the data collection issue. The machine learning models can be fed directly with extremely reliable data, and the results can be extracted.

2. Real data can be used to train the model(s). As a result, the efficiency and accuracy of models will improve, lowering the additional cost to the central authority.

3. The patient can get advise on how to live a healthy lifestyle. The model can be trained using advice given to other patients with similar problems or symptoms (by clinicians).

4. When a patient asks a simple question about his or her health, a trained model with a test is posted to the blockchain network; the model can predict outbreaks and make recommendations to doctors. Natural Language Processing can be used to diagnose an illness and make therapy recommendations.

5. The trained model can make clinical recommendations to clinicians based on the symptoms of the patient.

6. The model has been taught to forecast outbreaks. For example, if a patient has a test and the results are uploaded to the blockchain network, the model can predict an epidemic and make recommendations to the doctor.

7. To perform any medical test, we use a variety of machines and equipment in the healthcare industry. Every machine, or component of a machine, has a specific lifespan. It can also anticipate when the machine or a part of the machine will need to be changed or removed.

\section{Conclusions}

Recent advancement in using blockchain and Artificial Intelligence in Smart healthcare system drawn attention to researchers and developers. Internet of Medical Things researchers and developers are working together to merge different technology on large scale to benefit the society to the highest possible level. However, improvements are possible only if we consider various issues and shortcomings in the present smart technical approaches. In this research article, we design a system using two different technologies to enhance the efficiency and better results in Health care system. Our system have the ability to provide transparent, secure and intelligency in Internet of Medical Things (IoMT). 
Author Contributions: Conceptualization, M.H. and A.I.; investigation, M.H., A.I. and E.F. writingoriginal draft, M.H.; writing-review and editing, A.I. and R.P.; All authors have read and agreed to the published version of the manuscript.

Funding: Not applicable

Institutional Review Board Statement: Not applicable

Informed Consent Statement: Not applicable

Data Availability Statement: Data sharing is not applicable for this article.

Conflicts of Interest: The authors have no affiliation with any organization with a direct or indirect financial interest in the subject matter discussed in the manuscript.

\section{References}

1. Hassan, M.M.; Jincai, C.; Iftekhar, A.; Cui, X. Future of the Internet of Things Emerging with Blockchain and Smart Contracts. International Journal of Advanced Computer Science and Applications 2020, 11.

2. Kumar, S.; Tiwari, P.; Zymbler, M.L. Internet of Things is a revolutionary approach for future technology enhancement: a review. Journal of Big Data 2019, 6, 1-21.

3. Ahmad, M.A.; Teredesai, A.; Eckert, C. Interpretable Machine Learning in Healthcare. 2018 IEEE International Conference on Healthcare Informatics (ICHI) 2018, pp. 447-447.

4. $\quad$ spiderSilk - Home. https://spidersilk.com/. (Accessed on 10/28/2021).

5. Toh, C.; Brody, J.P. Applications of Machine Learning in Healthcare. 2021.

6. Jamison, D.T. Disease Control Priorities, 3rd edition: improving health and reducing poverty. The Lancet 2018, 391, e11-e14.

7. Zarzaur, B.; Stahl, C.C.; Greenberg, J.A.; Savage, S.A.; Minter, R.M. Blueprint for Restructuring a Department of Surgery in Concert With the Health Care System During a Pandemic: The University of Wisconsin Experience. JAMA surgery 2020.

8. He Warned of Coronavirus. Here's What He Told Us Before He Died. - The New York Times. https://www.nytimes.com/2020/0 2/07/world/asia/Li-Wenliang-china-coronavirus.html. (Accessed on 10/28/2021).

9. Wendelboe, A.M.; Miller, A.; Drevets, D.A.; Salinas, L.; Miller, E.J.; Jackson, D.; Chou, A.F.; Raines, J. Tabletop exercise to prepare institutions of higher education for an outbreak of COVID-19. Journal of emergency management 2020, 18 2, $183-184$.

10. Fekih, R.B.; Lahami, M. Application of Blockchain Technology in Healthcare: A Comprehensive Study. The Impact of Digital Technologies on Public Health in Developed and Developing Countries 2020, 12157, 268 - 276.

11. Best EMR Software Company | Medical Software I EHR I IMS | Meditab. https://www.meditab.com/. (Accessed on 10/28/2021).

12. Gagan Kumar, R.K. A survey on machine learning techniques in health care industry. International Journal of Recent Research Aspects 2016, 3, 128-132.

13. Gumaei, A.; Hassan, M.; Alelaiwi, A. A Hybrid Deep Learning Model for Human Activity Recognition Using Multimodal Body Sensing Data. IEEE Access 2019, 7, 99152-99160. doi:10.1109/ACCESS.2019.2927134.

14. Souri, A.; Ghafour, M.; Ahmed, A.; Safara, F.; Yamini, A.; Hoseyninezhad, M. A new machine learning-based healthcare monitoring model for student's condition diagnosis in Internet of Things environment. Soft Computing 2020, 24. doi:10.1007/s00500-02005003-6.

15. Ali, F.; El-Sappagh, S.; Islam, S.M.R.; Kwak, D.; Ali, A.; Imran, M.; Kwak, K. A Smart Healthcare Monitoring System for Heart Disease Prediction Based On Ensemble Deep Learning and Feature Fusion. Information Fusion $2020,63$. doi:10.1016/j.inffus.2020.06.008.

16. Chui, K.; Alhalabi, W.; Pang, S.; Pablos, P.; Liu, W.; Zhao, M.; Moraunti, L. Disease Diagnosis in Smart Healthcare: Innovation, Technologies and Applications 2017.

17. scikit-learn: machine learning in Python — scikit-learn 1.0.1 documentation. https://scikit-learn.org/stable/. (Accessed on 10/28/2021).

18. sklearn.ensemble.RandomForestClassifier — scikit-learn 1.0.1 documentation. https://scikit-learn.org/stable/modules/ generated/sklearn.ensemble.RandomForestClassifier.html. (Accessed on 10/28/2021).

19. Blockchain Applications in Healthcare. https://www.news-medical.net/health/Blockchain-Applications-in-Healthcare.aspx. (Accessed on 10/28/2021). 